\title{
Interference Diagnosis in Wireless Systems by using NI USRP
}

\author{
Athar Qureshi, Faculty of Engineering \& Science, \\ University of Greenwich, Chatham, United Kingdom \\ qm05@gre.ac.uk
}

\begin{abstract}
In both industrial and domestic environments, there will be a large increase of wireless communication systems. The density of wireless devices and need of additional bandwidth is expanding which is increasing the wireless interference. Interference emerges from unintended radiators is the main cause of degrading the Electromagnetic Compatibility and wireless communication performance in near field region and far field region and decrease the robustness and reliability. This paper presents methods of detection and removal of interference to improve EMC performance and wireless communication. We used NI USRP equipment for the diagnosis and provided the solutions to remove interference. The presented methods of interference diagnosis can efficiently optimize the performance of any wireless network.
\end{abstract}

Keywords- NI USRP, EMI, EMC, RFID, Bluetooth, NFC, Zigbee, Wifi, Wimax, GSM (2G), UMTS (3G), LTE (4G)

\section{INTRODUCTION}

Wireless communication can be defined as the transfer of information over electromagnetic waves. In both industrial and domestic environments, there will be a large increase of wireless communication systems. In next years, wireless will be a most important technology to enable the Internet of Things (IoT), Machine to Machine Communication (M2M) and Industry 4.0. Besides this, there is also increased use of sensor networks and cellular mobile networks. Wireless communication is the most crucial and fast growing technology of the world. From EU only in UK, only the numbers of mobile phone devices are 85 million. If we include other wireless devices, it would be in the range of half of billion. From TV remote to satellite communication the number of wireless devices increasing enormously. Cellular mobile phone always operates on very low power but their cumulative effects of interference are increasing with the growing numbers of users. Also with smaller base station cell size the aggregate effect of interference is also intensifying. Abnormal usage of the systems, age of the wireless equipment, global climate change, special interactions among base-station and its immediate environment are some of the other factors that escalating the interference. Also with the increase in bandwidth (data) and transmitting power requirement, the amount of interference is increasing. In short, smaller size of the systems and the increasing density of devices causing interference issues. Interference will be an expanding problem as new wireless systems are being introduced. For example, next generation cellular systems uses "femtocells" operating in an overlay network may potentially create interference to the macro-cell downlink.

Next generation wireless systems development raises two main problems: The first problem is the co-existence of all these systems, which can be solved by standardization. The second problem is the interference from unintended radiators, making wireless communication more vulnerable. In this work, we have presented the methods of diagnosis/detection of interference to enhance the robustness of communication from external interference at various commonly used wireless frequencies. This work also explores the intrinsic nature of interference which would provide practical industrial solutions to design Electromagnetic Compatibility (EMC) and management of wireless interference. Electromagnetic Compatibility (EMC) is related to near field interference of the wireless devices. It can be defined as the operations of low radiative electronic communication devices in the presence of high electromagnetic radiations.

A Simple Wireless Communication System is shown in the Figure 1. It consists up of the following:

1. Coded information is modulated into in-phase (I) and quadrature (Q) signals at baseband/intermediate frequency (IF) signal. This is known as IQ sampling.

2. Baseband further upconverted to Carrier or high frequency (HF) and wireless signal transmitted from Tx-Antenna through wireless channel.

3. Carrier signal receive at $\mathrm{Rx}$-Antenna and downconverted to baseband/ IF level.

4. I and Q component are separated, demodulated and decoded the information.

There are many types of modulation schemes in wireless communication. The encoded information may be modulated into some of the following modulation schemes at baseband IF level.

i. Amplitude Modulation

ii. Phase Modulation

iii. Frequency Modulation

iv. Pulse Width and Pulse Distance Modulation (PWM and PDM)

v. Pulse Amplitude Modulation (PAM) 
vi. Pulse Position Modulation (PPM)

In addition to IQ sampling, the amplification and filtration of the signal is also performed at IF frequency level both in transmitter and receiver. To transmit data for longer distance and to increase the frequency band separation, signal upconverted to $\mathrm{HF}$ carrier. Multiplexing can also be performed at this stage, in the case of multiuser communication systems. Then the antenna transmits information by propagation of electromagnetic waves through wireless channel. During the propagation of unwanted signals (interference) adds to the information carrying electromagnetic radiations and destroy the transmitted information. The aim of optimum wireless communication is to minimize the effects of interference so that useful transmitted information can be retrieved at the receiver. There can be many types of wireless channels types or channel models. The amount and nature of wireless interference is not the same in different channels. Hence, various types of wireless channels impact on the signal differently [1][2]. For example, urban wireless channel would be different compared to line of sight long distance communication due to more multipath wireless communication in urban environment. Therefore, different wireless channel models are important to design specific receiver for the wireless communication. On wireless receiver signals reach through multiple paths and at different time intervals cause delay spread. This causes one of the specific type interference known as intersysmbol interference. In higher modulation schemes this delay spread is more obvious which can be overcome by channel estimation and equalisation. In this paper for the simplicity of experimentation, we considered lower modulation schemes and neglect the effect of intersymbol interference. Multipath propagation also causes many other types of far field interferences that mentioned earlier including co-channel interference, adjacent channel interference and common mode interference. EMI affects the wireless signals only at near far region near to transmitter. Their impact can be very severe if EMC of the devices not properly managed. In industries, it is common practice to periodically test the EMI from the electrical and electronic devices for the raised interference.

At the receiver carrier signal received, amplified and downconverted to IF frequency level, reconstructed and filtered. Then IQ components separated. The information from IQ is retrieved and decoded. Reconstruction of the signal is to remove the wireless channel impacts on the wireless signal so that original information can be retrieved accurately. Multi access schemes like code division multiplexing access also support to mitigate interference but in reality the production of orthogonal codes for multiple access is a trivial problem. To retrieve the information accurately we may also use error control coding on the communicated information data. In addition to the above for wireless communication, we usually encrypt the data before sending and decrypt the data after receiving for the security of the communicated information. But this is not mandatory and depends on the wireless communication application. For example, for the patient X-
Ray imaging we don't required encoding, whereas, cellular mobile systems use advance encryption standard for information security.

Interference is defined as unwanted signal that adversely affects the wireless Communication. The interference source can be both internal and external. Some of the different types of wireless interferences are as follow:
1. Electromagnetic Interference (EMI)
2. Co channel Interference
3. Adjacent channel Interference
4. Intersymbol Interference
5. Inter carrier Interference
6. Common mode Interference

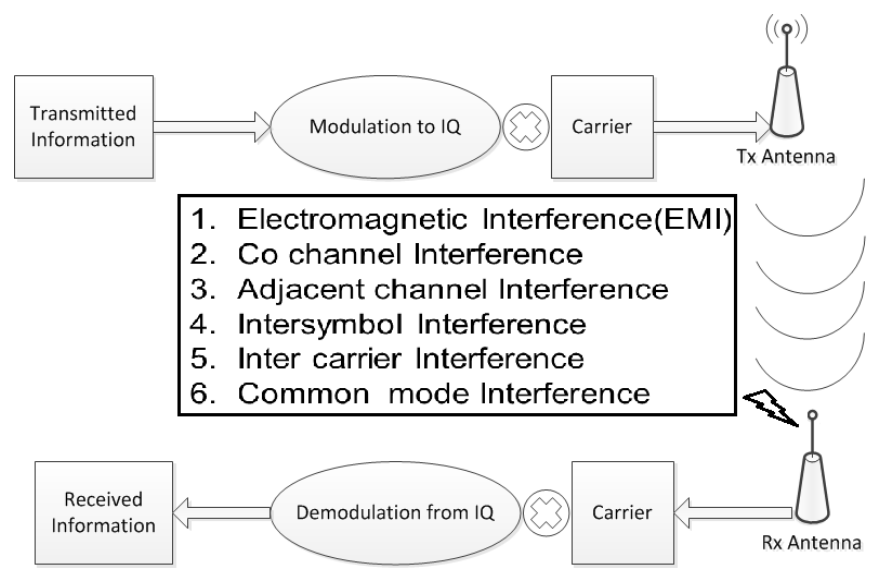

Figure 1. Simple Wireless Communication with Interference

Electromagnetic Compatibility (EMC) of electrical and electronic devices is the study that analyses the operations of low radiative electronic devices in high environmental radiations or electromagnetic interference. EMC is related to wireless interference in near field region. We can define Electromagnetic Compatibility (EMC) in simple words as the study of fine gadgets that operates in harsh industrial environment. In technical words Electromagnetic Compatibility (EMC) of the devices is the application that analyze the operations of low radiative electronic devices in high industrial interference. EMC is a characteristic of electrical and electronic equipment that allow it to operate in the presence of other electrical and electronic equipment, and not to adversely interfere with the other equipment. EMC has two aspects emission and susceptibility. All of the electrical and electronic equipment emits electromagnetic radiation energy, and some of that emitted energy may interact and interfere with other equipment. Equally, equipment may be susceptible to receiving energy emitted from other sources. Sometime wireless communication intentionally blocked by generating interference from intruder and create information security issues. The experiments presented in the paper are also useful to find any jamming of the communication. Apparently, radio transmitters and receivers are intended to 
emit and receive electrical energy, but other equipment may not be intended to do so. Even transmitters and receivers may emit and receive unwanted energy that may prevent those devices, or others, from functioning as intended. It is aim of the EMC to design and operate equipment so that it is both prevented from emitting spurious energy that can cause interference, and is immune to the adverse effects of any spurious energy that it may receive. Increasing bandwidth, number of Wireless systems, industrial vibrations, mechanical motion, leakage of the current, electrical contacts, hysteresis and reflections can cause EMI and interference, decrease wireless communication performance and create interference issues. There are generally two types of EMI that effects on EMC and wireless communication in near field region communication region:

1. Narrow Band EMI (Normally from other Radios and Reflectors)

2. Broad Band EMI (From Machineries, High Power Transmission etc).

Wireless communication and EMC has been comprehensively discussed in previous literature [2][3][4]. In next session we discuss NI USRP equipment [5] and test Narrow Band EMI and interference impact on wireless communication. In principal, it is equally valid for Broadband interference.

\section{SOFTWARE DEFINED RADIO, NI USRP}

We used the National Instrument USRP equipment known as Software Defined Radio (SDR) which has been used successfully for various wireless communication lab experiments in the previous literature [6][7][8][9][10]. The other radio test equipment like Agilent and Anritsu equipment etc can also use to measure interference to perform the same interference measurements. This paper has used an experimental setup to diagnose/detect interference in various wireless communication systems given in Table 1. Figure 2 shows the used NI USRP equipment. We used five different NI USRP modules to cover all frequencies ranges commonly used wireless communication. The NI USRP has two basic components: the NI USRP Radio System and NI USRP software driver based on NI LABVIEW Software. NI USRP provides programmer friendly tools to reduce the application development cost and time.

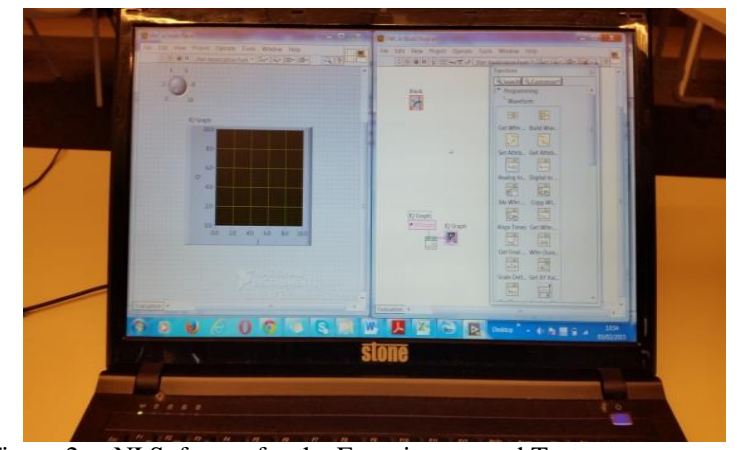

Figure 2a. NI Software for the Experiments and Tests

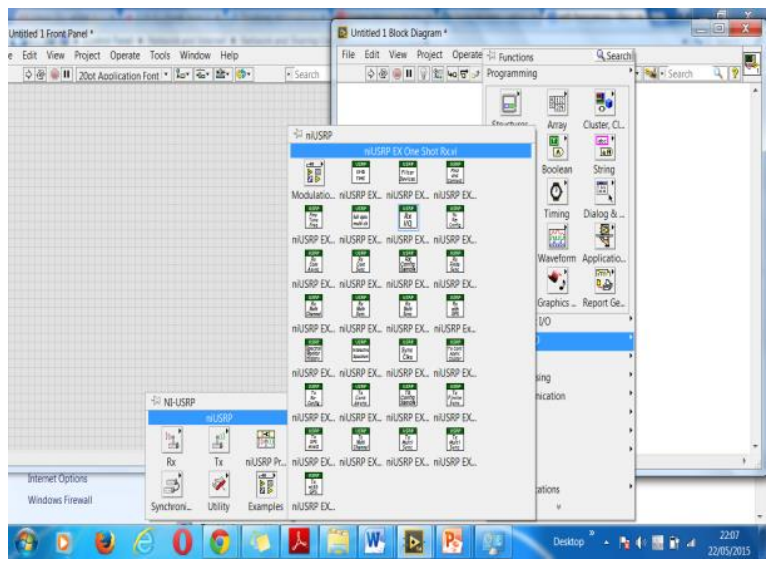

Figure 2b. NI USRP Software Driver for the Experiments and Tests

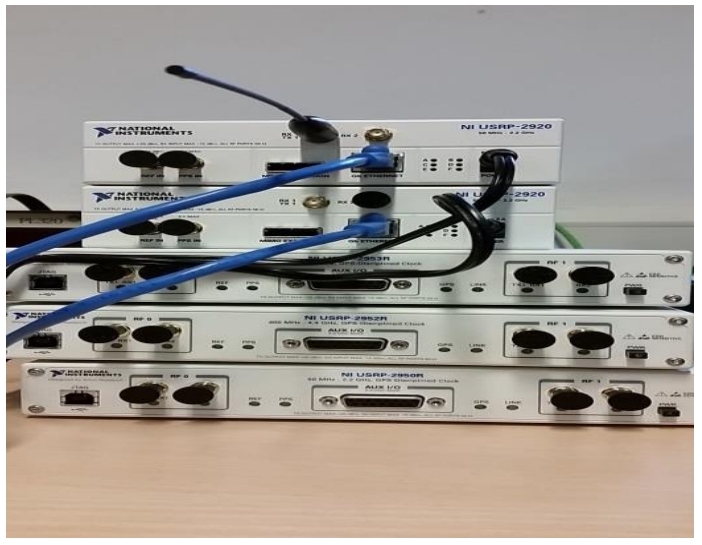

Figure 2c. NI USRP Hardware Equipment for the Experiments and Tests

\section{EXPERIMENTAL SETUP (TEST-BED)}

Figure 2a,2b, 2c shows the actual software and hardware equipment used in our experiments and Figure 3 shows the configuration of the experimental setup/test-bed for testing the interference and robustness under various wireless communication algorithms. It consists of NI USRP software and NI LABVIEW hardware. NI USRP is a programmable radio device. The test bed represents a simple wireless communication system shown in the Figure 1 . The configuration of the test-bed is shown in Figure 3 and it basically consists of wireless communication:

1. Transmitter (Tx)

2. Receiver $(\mathrm{Rx})$

3. Interference Signalling Source

NI USRP Software generates test signal, they are IQ modulated at baseband IF level and send over a HF carrier level from first NI USRP device as shown in Figure 3. On transmitter Tx the antenna gain kept low in compliance of EMC standards and to keep least disturbance to other wireless systems in near vicinity. Wireless carrier signal carry the information and travel through the wireless channel reach on the second NI USRP receiver Rx where HF carrier down 
converted to IF baseband level, IQ demodulated and information is recovered. We only consider the carrier wave to keep our experiments simple as we are mostly interested in the interference diagnosis in frequency domain. We kept antenna gain high at receiver ' $\mathrm{Rx}$ ', same as normally in practical wireless receivers. Third NI USRP equipment sends a narrow band interference signal of near carrier frequency ranges. For the simplicity the interference kept sinusoidal. All of these steps observed by NI USRP software plots in frequency domain and time domain on all three devices. We used NI USRP software spectrum analyzer for frequency domain and time domain measurement for the amplitude and frequency on second NI USRP 'Rx' device. We performed various experiments by increasing and decreasing antenna gain and distances on all of the three NI USRP devices. This test-bed represents a prototype of wireless communication in an industrial environment with interference.

For the simplicity, interference signal assumed to be periodic, continuous and reached on receiver asynchronously. It represents the narrow band interference from external devices. In practice this could be random and broadband which also can be detected with this experimental setup. Mathematically transmitted carrier signal is given in time domain

$x_{C}(t)=A_{C} \cos \omega_{C} t$

And interference signal is given by $\sum \mathrm{I}$.

By the superposition of interfering signal and carrier signal, the mathematical equation of the received signal is given as:

$x(t)=A_{C} \cos \omega_{C} t+\sum I$

Let we assume that external interference is sinusoidal then above equation can be written as:

$x(t)=A_{C} \cos \omega_{C} t+A_{I} \cos \omega_{I} t$

And consider that it has the same phase as of the carrier then above equation becomes:

$x(t)=A_{C} \cos \omega_{C} t+A_{I} \cos \omega_{C} t$

Simplifying the above yields:

$x(t)=\left(A_{C}+A_{I}\right) \cos \omega_{C} t$

The above equation clearly shows that the amplitude of the received signal would be summation of the carrier and interference signal and consequently increase the amplitude of the received signal. Therefore, unexpected amplitude of the received signal also represents the presence of the interference. The result is consistent with our experiments in next sessions and appendices.
The aim of the diagnosis experiments is to analyzed and view the first term and second term of the above equation separately. The above equation shows that the amplitude of both transmissions could be added and it is consistent with experimental results given in Appendices that shows with external interference can increase the amplitude of the signal when it is in phase with the carrier signal. In practical wireless systems this type of interference is very common called intermodulation interference. We increase and decrease the gain of the received signal and transmitted signal to recognize the received information carrying signal and interference by keeping interference signal constant. If first term and second term of equation 3 orthogonal then we can separately analyze both received signal and interference separately but in practice orthogonal codes are extremely difficult to produce and interference signal destruct the received signal and information. Particularly, from interference from external source always destroy the received signal if both lies on same frequency ranges.

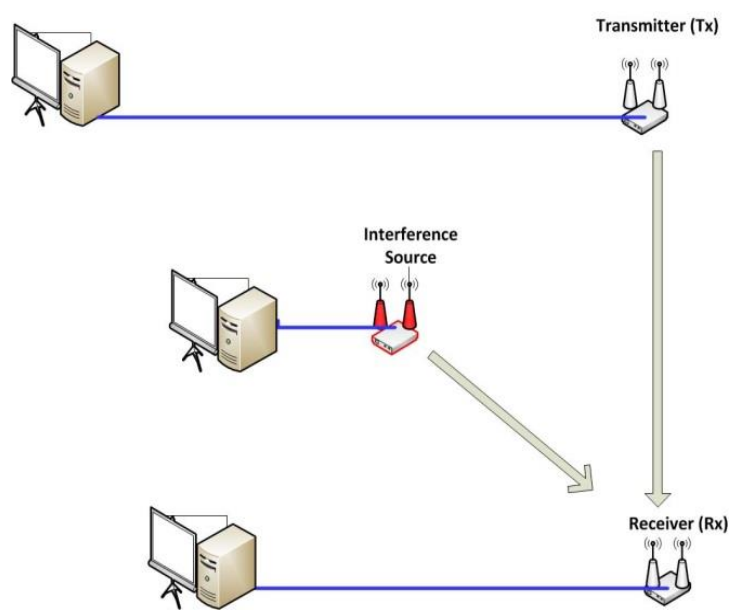

Figure 3. Experimental Configuration

\section{EXPERIMENTAL MEASUREMENT AND RESULTS}

Table 1 represents some of the commonly used industrial wireless systems standards and their frequency ranges. We carried out various experiments on these wireless communication. Some of the results are shown in the Appendices. In the experimental result we examined the $800 \mathrm{MHz}, 400 \mathrm{MHz}, 68-69 \mathrm{MHz}, 1.9 \mathrm{GHz}, 2.2 \mathrm{GHz}$ wireless communication between two NI USRP Tx and Rx. We send the test interference signal of near frequency ranges of wireless communication from a third NI USRP. And we observed the spectrum by amplitude-frequency and amplitudetime plots on the NI USRP Rx. We increased and decreased the gain of transmit and receive signal to recognize the communicated signals and interference. This setup can precisely diagnose any interference in industrial environment. 
This test-bed is prototype of industrial wireless communication and ready to test EMI and interference in any in industrial environment. As earlier discussed that EMI and interference can be of two types: Narrow band and wide band in near field region and far field region. We are able to detect both of the types by using this type of setup. With the help of this test-bed we can diagnose narrow band interference, random interference, wideband interference and propose a suitable wireless communication systems and transmission power for the specific industrial environment. Even wide band and random interference can be analyzed on the NI USRP software frequency-amplitude plot with same setup. This experimental setup can diagnose other types of far field interferences mention in section I and affectively identify the interference source. We can also adjust transmitting and receive power of the devices according to the communication requirement. As discuss earlier that unnecessary transmitting power can produce destructive effects on other wireless devices in the vicinity. By using this test-bed we can recommend appropriate power of communication signals. In our experiments we analyzed only sinusoidal interference impact on the communication but this method can also observe other types of interferences by programming the third device to generate different types of interference signals or with industrial wireless interference source. We have performed various experiments in the presence of narrow band interference and observed the variation on the received signal when we shifted the interference signal at various positions in the range of communication frequency to diagnose interference. The EMI and interference is already presented in previous research literature with some of the practical demonstration [11][12][13][14][15] but we found that detailed practical were missing in previous literature, particularly with NI USRP equipment. Also the theoretical analysis already has been presented in previous literature [16][17][18].

Observed experimental results provide the complete pictures of constructive and destructive interference pattern in time domain. It is also observed that whenever interference signal impacting severely the communication information signals, we observe increase in the amplitude of the signal in frequency domain. Unusual signal amplitude, more than the expected amplitude of received signal also reveals the presence of interference in wireless communication. Therefore, when conducting the EMC and interference test of the factories, we should keep in mind that un-usual amplitude or abnormal amplitude of the Rx signal some time represents the presence of interference and we have to check more magnified view of the signal in frequency domain and time domain to precisely observed the interference and actual received signal. We should also shift the communication frequency to have better picture of interference. Closer the interference signal band to the communication signal provide clearer pattern of interference in time domain. As expected that if we shift the interference signal far from the communication frequency, less affects would be on the communication and we should always observe in both plots in frequency domain and time domain. NI USRP plots in time and frequency domain provide clear picture of the quality of communication. We have shown the experimental results in appendices. In case of the interference from a broad band signal, we would see a clear picture $\mathrm{Rx}$ amplitude and interference signal.

\begin{tabular}{|l|l|l|l|}
\hline $\begin{array}{l}\text { Wireless } \\
\text { Systems }\end{array}$ & Frequencies & $\begin{array}{l}\text { Wireless } \\
\text { Systems }\end{array}$ & Frequencies \\
\hline NFC & $13.56 \mathrm{MHz}$ & Zigbee & $868 \mathrm{MHz}$ \\
\hline RFID & $433 \mathrm{MHz}$ & $\begin{array}{l}\text { GSM (2G) } \\
\text { AMPS }\end{array}$ & $400 \mathrm{MHz}$ \\
\hline RFID & $865 \mathrm{MHz}$ & GSM (2G) & $900 \mathrm{MHz}$ \\
\hline Wifi & $2.4 \mathrm{GHz}$ & GSM (2G) & $1800 \mathrm{MHz}$ \\
\hline Wimax & $2.3 \mathrm{GHz}$ & UMTS(3G) & $2100 \mathrm{MHz}$ \\
\hline Bluetooth & $2.16 \mathrm{GHz}$ & LTE(4G) & $800 \mathrm{MHz}$ \\
\hline Zigbee & $2.4 \mathrm{GHz}$ & LTE (4G) & $2600 \mathrm{Mhz}$ \\
\hline
\end{tabular}

Table 1. Used Wireless Standards and Frequencies in Experiments

\section{CONCLUSIONS}

We have presented a test-bed to examine EMI in wireless communication systems. We can improve the EMC performance, diagnose interference and optimize wireless communication by using the developed test bed in any industrial environments. The developed test-bed is also useful for developing wireless communication systems and devices for the Industry.

We have performed various experiments on the developed test-bed. The experiment shows that higher the amplitude of Rx signal than normal could be the presence of EMI and constructive interference. We have presented several experiments on industrial wireless communication systems shown in Appendices. Our experiments reveal that to improve the performance of Wireless Communication (RFID, NFC, Bluetooth, Wifi, 2.5G, 3G, $4 \mathrm{G}$ etc.) we need to perform following steps:

Step 1. Adjust the power of wireless devices, Observe and keep the record of the receive spectrum of communication Devices in Anechoic Chamber or in EMI and interference free environment (Normal Spectrum).

Step 2. Switched off the Industrial Machines and insulate the possible sources of EMI/interference and observe the Receive Spectrum, it should be close to the observation and according to the record of the Step 1.

Step 3. Switched on the Industrial Machines and observe the Spectrum. Keep repeating Step 2 until all necessary shielding 
made on the possible sources of interference. And all the unwanted source of interference removed.

NI USRP software provide the programming interface, it would be exciting to develop code to obtain interference graphs/plots of various kind other than frequency domain and time domain measurement and observation of the communication.

\section{ACKNOWLEDGMENT}

This research work is generously funded by i-MOSYDE, Cluster, INTERREG-IV Project and University of Greenwich, UK, from August, 2014 to September, 15. It was presented in the IMOSYDE Workshop, University of Greenwich, UK.

\section{REFERENCES}

[1] A. Goldsmith, Wireless Communication, Cambridge Univ. Press, 2005.

[2] Agilent Digital Modulation in Communications Systems. An Introduction Application Note 1298, USA 14, March, 2001.

[3] Electromagnetic Compatibility Aspects of Radio-based Mobile Telecommunications Systems, Link Collaborative Research Personal Communication Programme, Final Report.

[4] Vasquez, Horacio, et al. "Simple device for electromagnetic interference shielding effectiveness measurement", Institute of Electrical and Electronics Engineers. 2009.

[5] "An Introduction to Software Defined Radio With LabVIEW and NI USRP", NI USRP Resources.

[6] S.Costanzo, F,Spadafora, Borgia, H.O. Moreno, A. Costanzo, G. DiMansanza, High rsolution software defined radar system for target detection, Journal of Electrical and Computer Engineering, vol 2013, ANo 7, January 2013.

[7] Shahin, N, LaSorte, N.J. ; Rajab, S.A. and Refai, H.H, "802.11g channel characterization utilizing labview and NI-USRP", Instrumentation and Measurement Technology Conference (I2MTC), 2013 IEEE International, Minneapolis, USA, Pages, 753 -75, 6-9 May 2013.

[8] Samaan, and Alex Perdomo Elian, "Global Positioning System Signal Acquisition Using USRP”, Florida International University, Department of Computer and Electrical Engineering, April, 2014

[9] Kim, N., N. Kehtarnavaz, and M. Torlak. "LabVIEW Based Software-Defined Radio: 4-QAM Modem." framework 10 (2007): 11.

[10] Bruce A. Black, "Basic Communication Systems Lab Exercises using the NI-USRP, Rose-Hulman Institute of Technology, NI USRP Resource.

[11] Espino, J., Markendahl, J., "Analysis of macro-femtocell interference and implications for spectrum allocation,"IEEE 20th International Symposium on Personal, Indoor and Mobile Radio Communications, September 2009.

[12] Lui, P.L., "Passive intermodulation interference in communication systems," Electronics \& Communication Engineering Journal, June 1990.

[13] Betts, J.A., "Intermodulation interference in mobile multipletransmission communication systems operating at high frequencies (3-30 MHz)," Proceedings of the Institution of Electrical Engineers, November, 1973.

[14] Jabbar, M., Rahman, M, "Radio frequency interference of electric motors and controls," Conference Record of the 1989 IEEE Industry Applications Society Annual Meeting, October, 1989.
[15] 3GPP, "Technical Report: Feasibility Study for Evolved Universal Terrestrial Radio Access (UTRA) and Universal Terrestrial Radio Access Network (UTRAN)," 2011.

[16] Y. Patenaude, J. Dallaire, F. Menard, and S. Richard, "Antenna pim measurements and associated test facilities," IEEE Antennas and Propagation Society International Symposium, vol. 4, pp. 620-623, Jul. 2001.

[17] J. Henrie, A. Christianson, and W. Chappell, "Prediction of passive intermodulation from coaxial connectors in microwave networks," IEEE Trans. on Microwave Theory and Techniques, vol. 56, no. 1, pp. 209-216, Jan. 2008.

[18] S. Hienonen, V. Golikov, , P. Vainikainen, and A. Raisanen, "Near-field scanner for the detection of passive intermodulation sources in base station antennas," IEEE Trans. on Electromagnetic Compatibility, vol. 46, no. 4, pp. 661-667, Nov. 2004 


\section{APPENDICES}

Appendix 1:

$800 \mathrm{MHz}$ received carrier signals (2G GSM, 4G LTE, RFID, Zigbee)

Plot 1 represents amplitude-time plot (time domain)

Plot 2,3,4 are same plots in frequency-time(frequency domain)

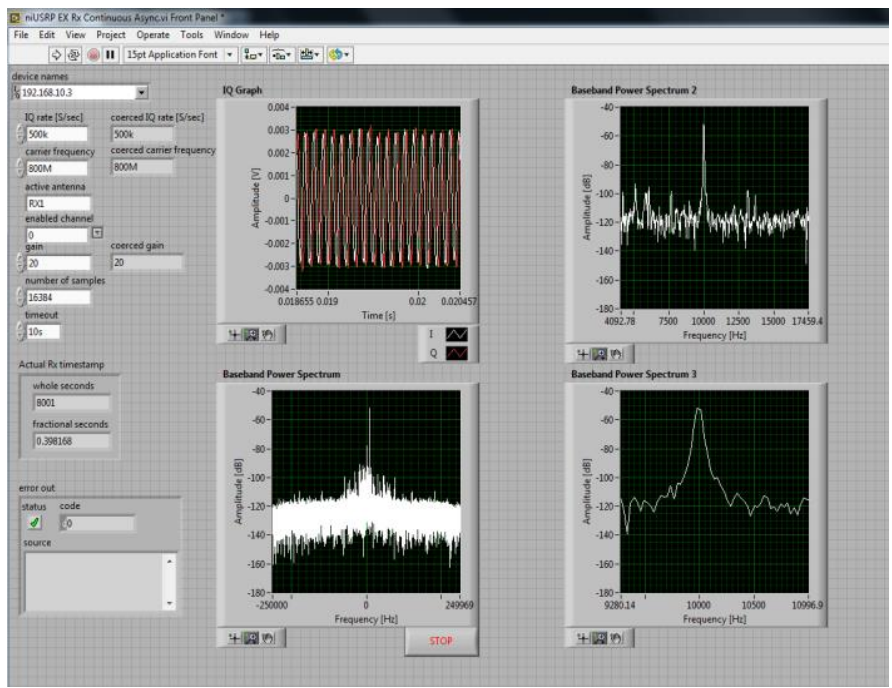

Figure 4. Carrier without Interference

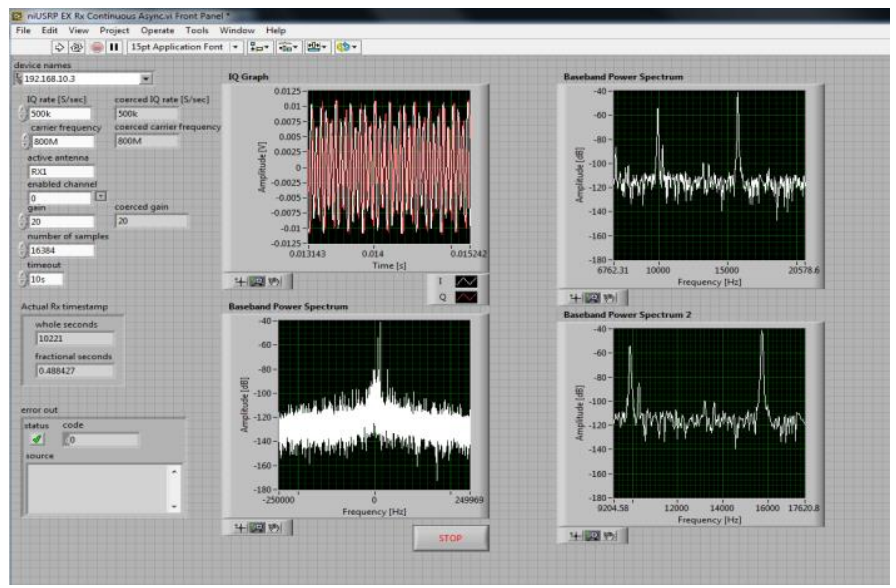

Figure 5. With Interference, the frequency band separation is $6000 \mathrm{~Hz}$ between carrier and Interference.

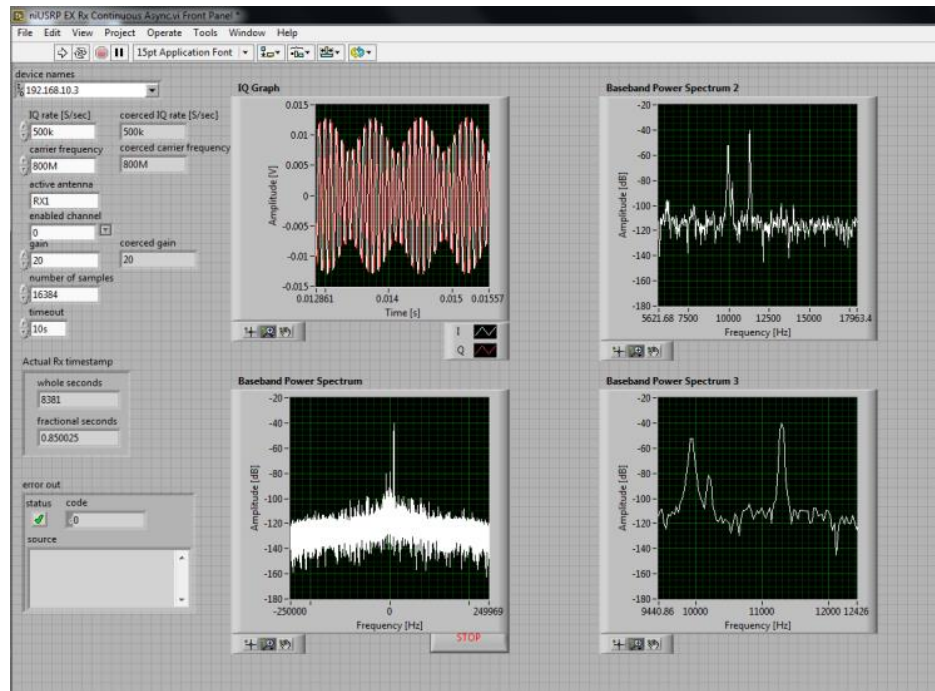

Figure 6. With Interference, the frequency band separation is $1200 \mathrm{~Hz}$ between carrier and Interference.

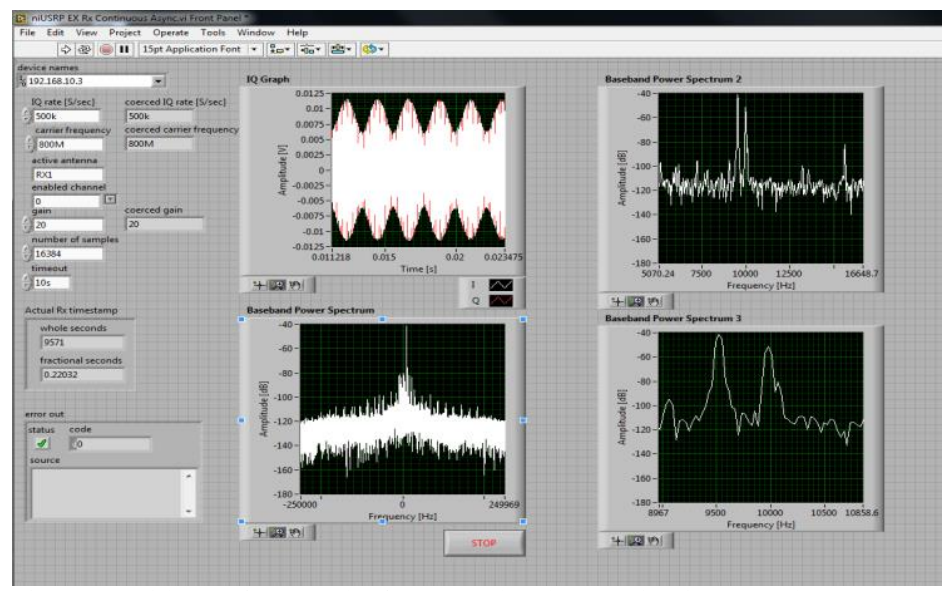

Figure 7. With Interference, the frequency band separation is $500 \mathrm{~Hz}$ between carrier and Interference.

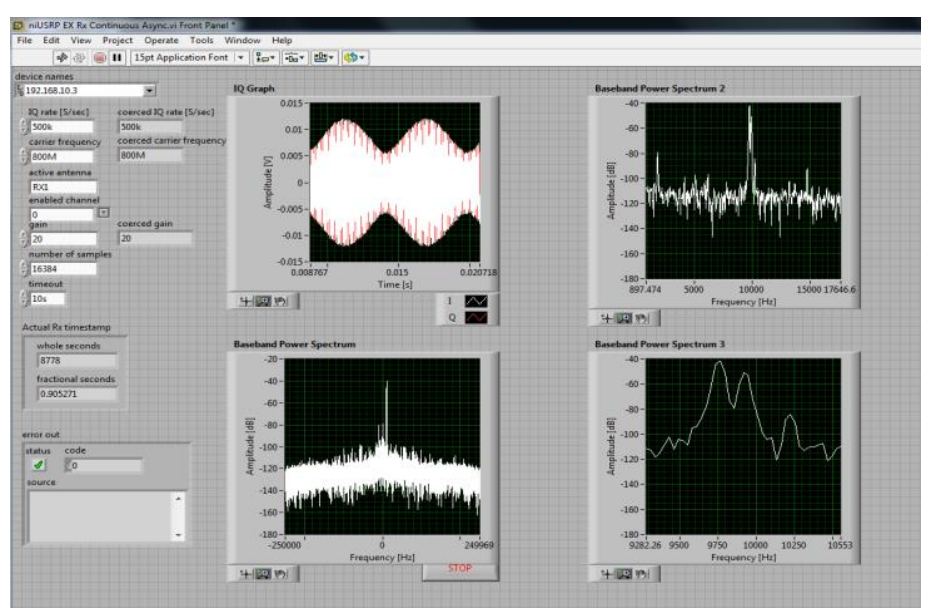

Figure 8 . With Interference, the frequency band separation is $25 \mathrm{~Hz}$ between carrier and Interference. 
Appendix 2:

$400 \mathrm{MHz}$ received carrier signals (Zigbee, $2 \mathrm{G} \mathrm{GSM}$,

$1 \mathrm{G}$ AMPS)

Plot 1 represents amplitude-time plot (time domain)

Plot 2,3,4 are same plots in frequency-time(frequency domain)

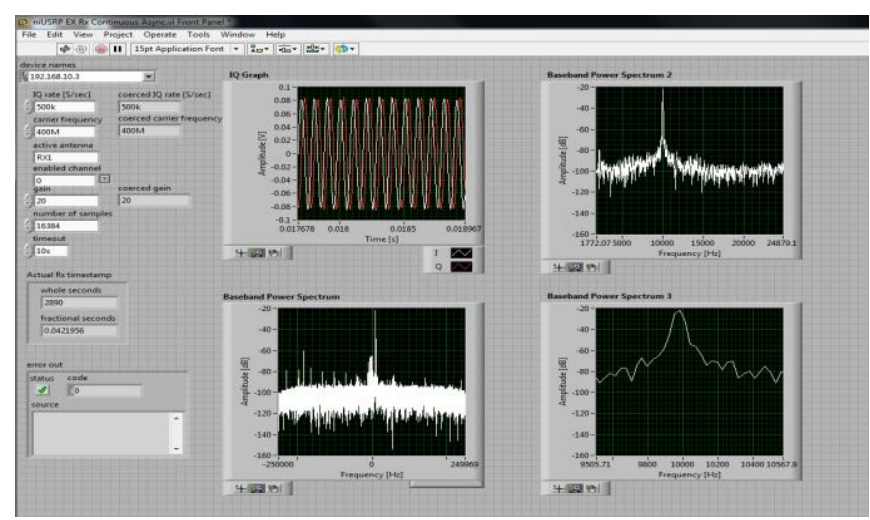

Figure 9. Carrier without Interference

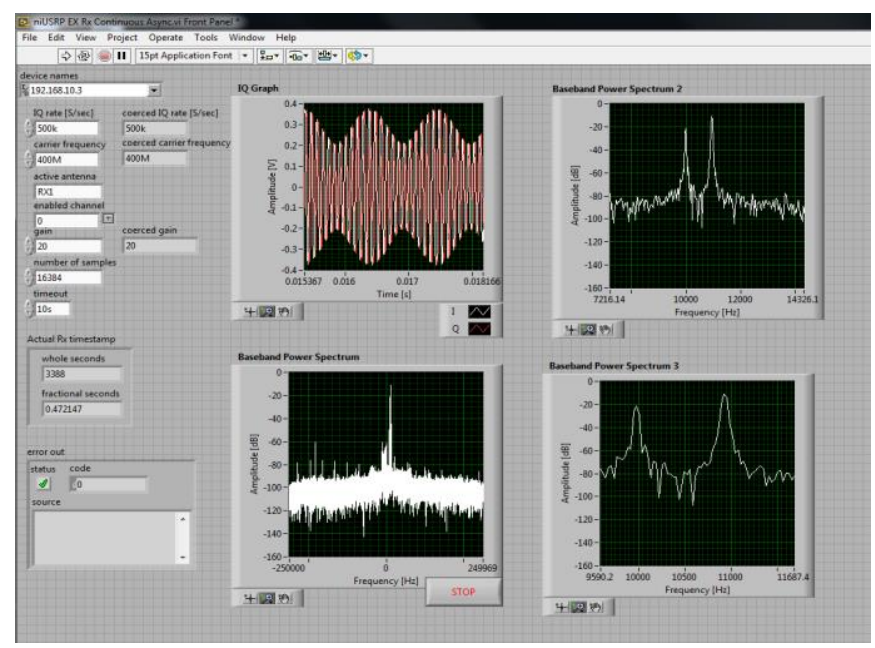

Figure 10. With Interference, the frequency band separation is $1000 \mathrm{~Hz}$ between carrier and Interference

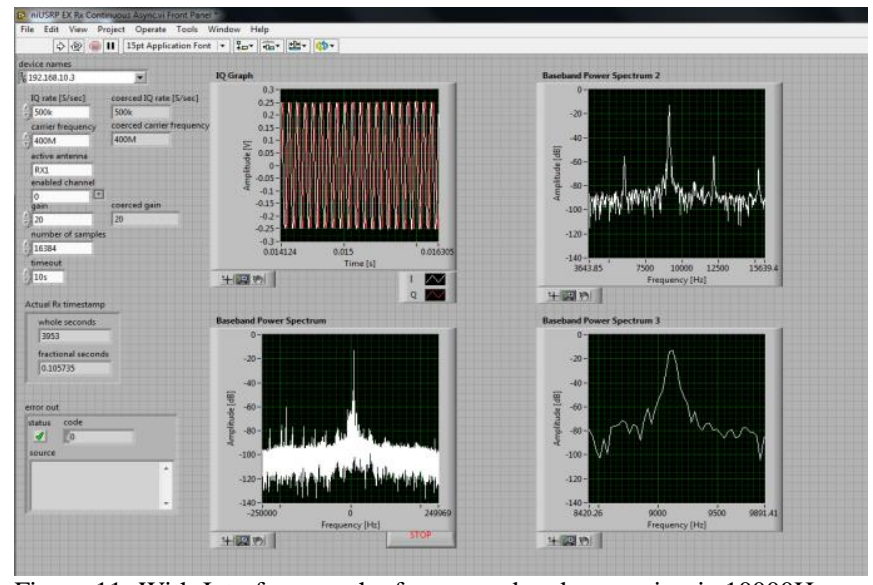

Figure 11. With Interference, the frequency band separation is $10000 \mathrm{~Hz}$ between carrier and Interference.
Appendix 3:

68-69MHz received carrier signals (NFC)

Plot 1 represents amplitude-time plot (time domain)

Plot 2,3,4 are same plots in frequency-time(frequency domain)

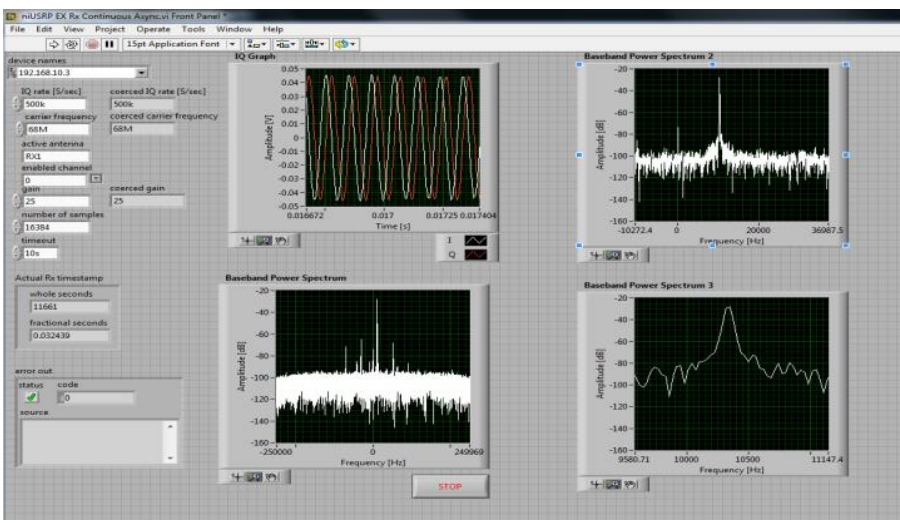

Figure 12. Carrier without Interference

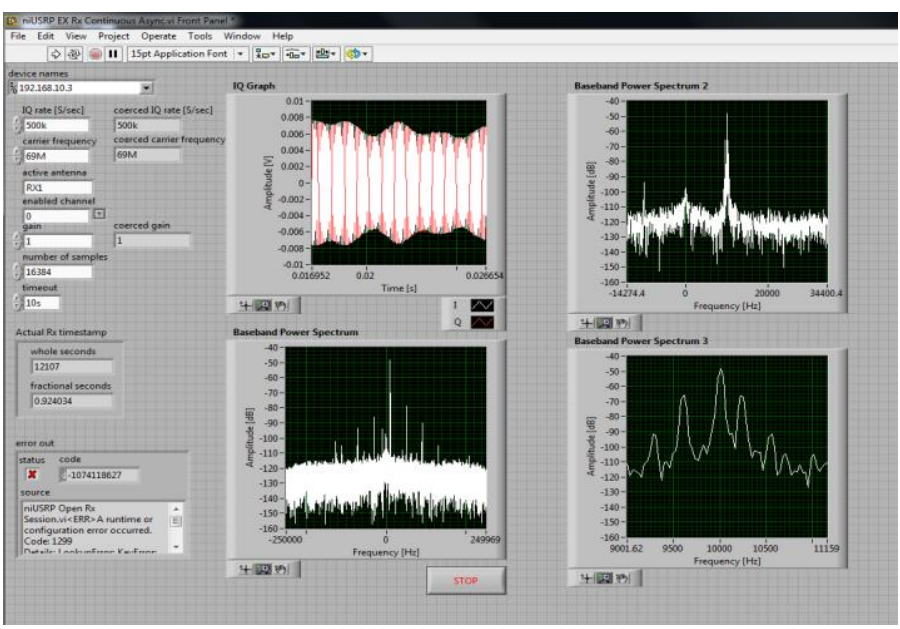

Figure 13. With four Interference from various environmental signal

Appendix 4:

2.2GHz received carrier signals (3G-UMTS, 4G-LTE, Wifi, Wimax,

Zigbee etc.)

Plot 1 represents amplitude-time plot (time domain)

Plot 2,3,4 are same plots in frequency-time(frequency domain)

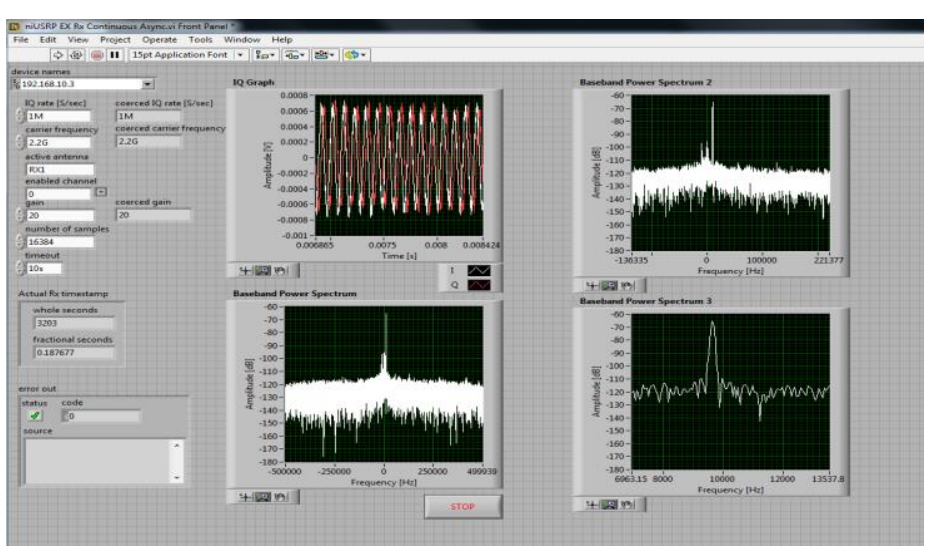

Figure 14. Carrier Without Interference 


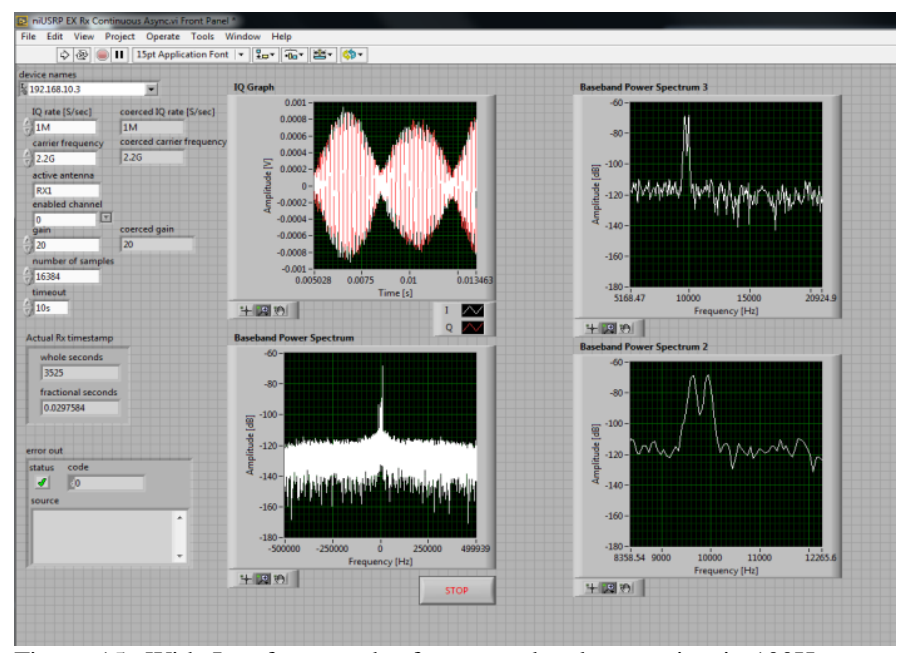

Figure 15: With Interference, the frequency band separation is $100 \mathrm{~Hz}$ between carrier and Interference.

Appendix 5:

$1.9 \mathrm{GHz}$ received carrier signals (2G-GSM, 3G-UMTS)

Plot 1 represents amplitude-time plot (time domain)

Plot 2,3,4 are same plots in frequency-time(frequency domain)

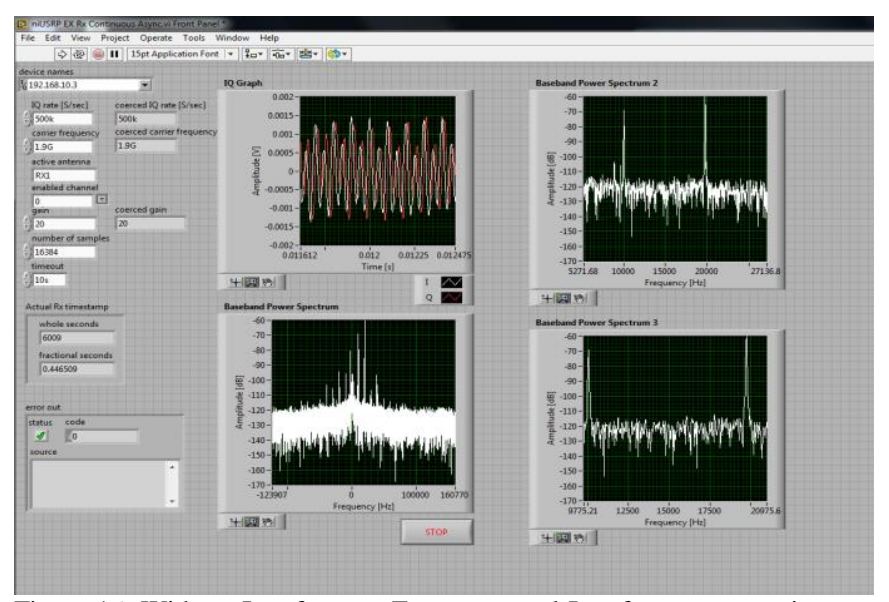

Figure 16. Without Interference. Frequency and Interference separation is broad and therefore, interference is not affecting the received signal.

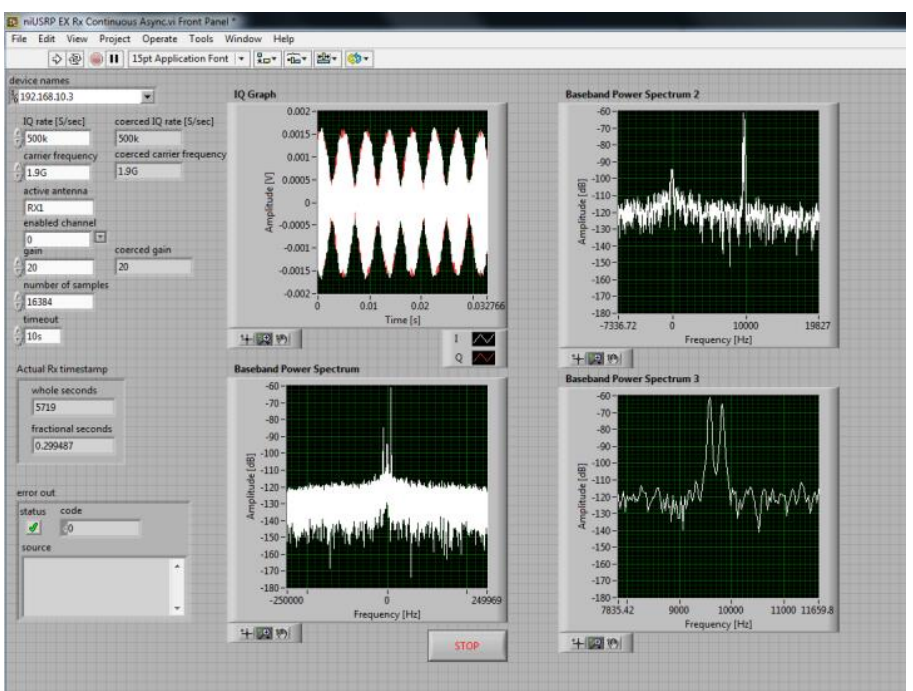

Figure 17. With Interference, the frequency band separation is $100 \mathrm{~Hz}$ between carrier and Interference.

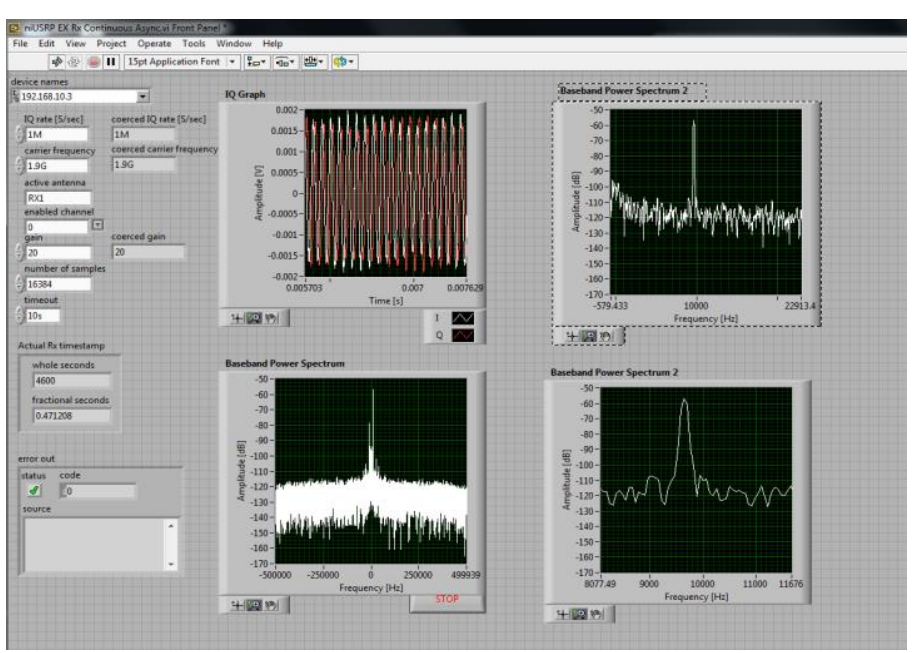

Figure 18. Without Interference. Frequency and Interference separation is broad and therefore, interference is not affecting the received signal. 